\title{
Microalbuminuria in Diabetic Patients in the Bamenda Health District
}

\author{
Marie Ebob Agbortabot Bissong ${ }^{1,2,3, ~ *, ~ G e r a l d ~ N g o ~ T e k e ~}{ }^{3}$, Moses Samje ${ }^{3}$, Harris Goneh ${ }^{1}$, \\ Frank Eric Tatsing Foka ${ }^{2}$, Henri-Lucien Kamga ${ }^{1}$ \\ ${ }^{1}$ Department of Medical Laboratory Sciences, University of Bamenda, Bambili, Cameroon \\ ${ }^{2}$ Department of Biological Sciences, North West University, Mafikeng, South Africa \\ ${ }^{3}$ Department of Biomedical Sciences, University of Bamenda, Bambili, Cameroon
}

Email address:

mabissong@yahoo.com (Marie E. A. B.),ngobob@yahoo.com (Gerald N. T.), msamje@yahoo.com (Moses S.), harrisgoneh@gmail.com (Harris G.), frankeric2023@yahoo.fr (Frank E. T. F.), henrikamga2002@yahoo.fr (Henri-Lucien K.)

*Corresponding author

\section{To cite this article:}

Marie Ebob Agbortabot Bissong, Gerald Ngo Teke, Moses Samje, Harris Goneh, Frank Eric Tatsing Foka, Henri-Lucien Kamga. Microalbuminuria in Diabetic Patients in the Bamenda Health District. Science Journal of Clinical Medicine.

Vol. 6, No. 4, 2017, pp. 63-67. doi: 10.11648/j.sjcm.20170604.14

Received: March 15, 2017; Accepted: April 19, 2017; Published: October 18, 2017

\begin{abstract}
Diabetic nephropathy (DN) is the most common cause of end stage renal disease (ESRD) and it accounts for one third of all patients requiring renal replacement therapy in Africa. Diabetic patients with microalbuminuria (MA) have an increased risk of progression to macroalbuminuria and later to ESRD. So detecting MA which is a marker of DN helps to alert the clinician to intervene at a time when future renal damage is still preventable. The main goal of this study was to determine the prevalence of MA in diabetic patients at the Bamenda Regional Hospital. This study was a prospective cross sectional study involving diabetic patients at the Bamenda Regional Hospital. A random (spot) or first morning urine specimen was collected and MA was measured by a semi quantitative dipstick method using the URS-14H urine test strips. The prevalence of MA in the present study was $34.6 \%$, which is high but similar to the results of other studies done in sub-Saharan Africa. Although MA was more common (50\%) in individuals with diabetes more than 16 years, a high proportion $(42.85 \%)$ of patients with MA had a duration of diabetes of $\leq 5$ years. There was no significant difference in the occurrence of MA with respect to age, gender and duration of diabetes. This study showed a high prevalence of MA in the diabetic population presenting at the Bamenda Regional Hospital. Consequently, measures of glycemic control should be enhanced in this population to prevent the progression to macroalbuminuria and ESRD.
\end{abstract}

Keywords: Microalbuminuria, Diabetes Mellitus, Diabetic Nephropathy, Prevalence, Biomarker

\section{Introduction}

Diabetes mellitus is a metabolic disorder characterized by chronic hyperglycemia with disturbances of carbohydrate, fat and protein metabolism resulting from defects in insulin secretion, action or both [1]. Long term complications of diabetes are associated with microvascular and macrovascular complications that can lead to visual impairment (retinopathy), blindness, kidney disease (nephropathy), nerve damage, amputation, heart disease and stroke [2]. Renal disorder is a common complication in diabetic patients and urinary microalbumin level is an important marker of chronic kidney disease and end stage renal disease (ESRD). Generally, nephropathy has been viewed as a descending path of normoalbuminuria (NA) to end stage renal disease (ESRD) through an intermediate stage marked by microalbuminuria [2].

Diabetic nephropathy (DN) is the main cause of morbidity and premature mortality in diabetic patients $[3,4]$. This complication is first manifested as microalbuminuria (MA). Diabetic patients with MA have an increased risk of progression to overt proteinuria and after sometime, renal failure $[5,6]$. Approximately $80 \%$ of type 1 diabetic patients with persistent MA develop overt nephropathy after 10- 
15years and 50\% later on develop ESRD. In type 2 diabetics $20-40 \%$ with microalbuminuria progress to overt nephropathy after 20years from onset and approximately $20 \%$ develop ESRD [6]. DN has been extensively studied and reported in the western world $[5,6,7,8,9]$ but information on nephropathy in African diabetic patients are scarce. In Cameroon, MA was detected in $53.1 \%$ of diabetic patients in Hospitals in Yaounde and high blood pressure and duration of diabetes were reported to be the leading confounding factors [3].

Microalbuminuria is a term used to describe a moderately increase in the amount of albumin in urine [3]. It can be defined as a urinary albumin excretion rate (UAE) of between $30-300 \mathrm{mg} / 24$ hour or as an albumin/creatinine ratio (ACR) of greater than $30-300 \mathrm{mg}$ albumin/g of creatinine [4]. The UAE or ACR tests are generally used for the screening of MA but in situations where these are not available, MA could be screened using the semi-quantitative tests such as Micral microalbumin urine test and Clinitec Microalbumin [10].

Diabetic nephropathy is the leading cause of ESRD worldwide and the third most common cause of chronic kidney disease in Africa after hypertension and glomerulonephritis [11]. It also accounts for one third of all patients requiring renal replacement therapies, which are very expensive and not widely available in Africa due to cost and lack of expertise [11]. The early detection of microalbuminuria is very important in the management of $\mathrm{DN}$ and in preventing the onset of ESRD. In the developing countries, this is even more important because recommended renal therapies such as transplants are not available probably due to economic constraints. Thus, screening for MA in diabetic patients remains paramount in the control of DN in most developing countries. Consequently, the present study was designed with aim to determine the frequency of microalbuminuria in diabetic patients presenting at the Bamenda Regional Hospital.

\section{Method}

\subsection{Study Design}

This study was a prospective cross sectional study involving diabetic patients presenting at the Bamenda Regional Hospital during the period of January to May 2016. BRH is one of the largest hospitals in the Northwest Region and being located in the chief town of the region, this hospital receives patients from all over the region and even beyond. Authorization for the study was granted by the Northwest Regional delegation for Public Health and the Department of Medical Laboratory Sciences of the University of Bamenda, Cameroon.

\subsection{Sampling}

A total of 100 healthy diabetic patients of age $\geq 18$ were recruited into the study after being consented. Participants who presented with signs and symptoms of UTI such as fever, dysuria and frequent urination and any underlying conditions were excluded from the study. At the end of the screening exercise, nineteen participants were rejected from the study.

\subsection{Data Collection}

A questionnaire including demographic data and medical history was completed by each participant. The height and weight of each participant was measured to estimate the body mass index (BMI) and a BMI $<25 \mathrm{~kg} / \mathrm{m}^{2}$ was considered to be normal $[10,12]$. The blood pressure of participants was recorded with the aid of a mercury sphygmomanometer in the right upper arm in the sitting position after five minutes rest by a trained personnel. Patients were categorized as hypertensive if the systolic blood pressure was $>130 \mathrm{mmHg}$ and / or diastolic blood pressure was $>85 \mathrm{~mm} \mathrm{Hg}[11,12]$.

\subsection{Laboratory Analysis}

Early morning urine samples were collected from each patient in a clean urine container and the dipstick microalbumin test was done using urine test strips (URS$14 \mathrm{H}$, England) and the procedures were carried out following the manufacturer's instructions.

\subsection{Statistical Analysis}

All data from this study were analyzed using SPSS (Statistical Package for Social Sciences) Version 20.0. Significant differences between categorical variables were determined using the Chi-Square test at a $95 \%$ confidence level. $P<0.05$ was considered statistically significant.

\section{Results}

A total of 100 diabetic patients enrolled into the study; out of these, 19 were excluded based on the exclusion criteria and we were left with 81 participants. The majority of the participants $(40,49.4 \%)$ were 60 years and above (Table 1 ). There were more females $(59,72.8 \%)$ in the study than males and most of the participants $(65,80.2 \%)$ live in urban areas.

The prevalence of microalbuminuria according to socioeconomic and demographic parameters is presented in Table 1. Out of the 81 participants 28 (34.6\%) had microalbuminuria while $53(64.4 \%)$ had normal albumin levels (normoalbuminuria). Despite the high prevalence of MA in female $(21,25.9 \%)$ than in male $(7,8.6 \%)$ diabetic patients, there was no significant difference in the occurrence of MA with respect to gender. Interestingly, none of the diabetic patients below the age of 40 years had MA. Furthermore, there was no significant difference in the occurrence of MA with respect to age, marital status, area of residence, level of education and monthly income $(\mathrm{P}>0.05)$. 
Table 1. Prevalence of microalbuminuria according to demographic parameters.

\begin{tabular}{|c|c|c|c|c|c|}
\hline \multirow{2}{*}{ Parameter } & \multirow{2}{*}{ Category } & \multicolumn{2}{|c|}{ Microalbuminuria } & \multirow{2}{*}{ Total } & \multirow{2}{*}{$P$ value } \\
\hline & & Absent (\%) & Present (\%) & & \\
\hline \multirow{3}{*}{ Age (years) } & $18-40$ & $5(6.2)$ & $0(0.0)$ & $5(6.2)$ & \multirow{3}{*}{0.17} \\
\hline & $41-60$ & $21(25.9)$ & $15(18.5)$ & $36(44.4)$ & \\
\hline & $>60$ & $27(33.3)$ & $13(16.0)$ & $40(49.4)$ & \\
\hline \multirow{3}{*}{ Gender } & Female & $38(46.9)$ & $21(25.9)$ & $59(72.8)$ & \multirow{3}{*}{0.75} \\
\hline & Male & $15(18.5)$ & $7(8.6)$ & $22(27.2)$ & \\
\hline & Single & $2(2.5)$ & $2(2.5)$ & $4(4.9)$ & \\
\hline \multirow[t]{2}{*}{ Marital status } & Married & $35(43.2)$ & $18(22.2)$ & $53(65.4)$ & \multirow[t]{2}{*}{0.80} \\
\hline & Widowed & $16(19,8)$ & $8(9.9)$ & $24(29.6)$ & \\
\hline \multirow{3}{*}{ Residence } & Rural & $9(11.1)$ & $7(8.6)$ & $16(19.8)$ & \multirow{2}{*}{0.39} \\
\hline & Urban & $44(54.3)$ & $21(25.9)$ & $65(80.2)$ & \\
\hline & None & $15(18.5)$ & $8(9.9)$ & $23(28.4)$ & \\
\hline \multirow{3}{*}{ Level of education } & Primary & $23(28.4)$ & $11(13.5)$ & $34(42.0)$ & \multirow{3}{*}{0.64} \\
\hline & Secondary & $10(12.5)$ & $8(9.9)$ & $18(22.2)$ & \\
\hline & Tertiary & $5(6.2)$ & $1(1.2)$ & $6(7.4)$ & \\
\hline \multirow{3}{*}{$\begin{array}{l}\text { Estimated monthly } \\
\text { income }\end{array}$} & $<90.000$ Frs & $40(49.4)$ & $21(25.9)$ & $61(75.3)$ & \multirow{3}{*}{0.89} \\
\hline & $90.000-150.000 \mathrm{Frs}$ & $10(12.3)$ & $6(7.4)$ & $16(19.8)$ & \\
\hline & $>150.000 \mathrm{Frs}$ & $3(3.7)$ & $1(1.2)$ & $4(4.9)$ & \\
\hline
\end{tabular}

The frequency of microalbuminuria was compared with some behavioral and biochemical markers as shown in Table 2. Although MA was more common $(6,50 \%)$ in individuals with diabetes for more than 16 years than those with duration of diabetes less than 16 years, this difference was not statistically significant. Also, $12(42.85 \%)$ of patients with MA had a duration of diabetes of $\leq 5$ years. In addition, there was no significant difference in the frequency of MA with respect to the history of smoking, body mass index (BMI), systolic blood pressure and diastolic blood pressure $(\mathrm{P}>0.05)$.

Table 2. Correlation of microalbuminuria and some risks factors.

\begin{tabular}{|c|c|c|c|c|c|}
\hline \multirow{2}{*}{ Parameter } & \multirow{2}{*}{ Category } & \multicolumn{2}{|c|}{ Microalbuminuria } & \multirow{2}{*}{ Total } & \multirow{2}{*}{ p-value } \\
\hline & & Absent (\%) & Present (\%) & & \\
\hline \multirow{4}{*}{ Duration of diabetes (years) } & $\leq 5$ & $23(65.7)$ & $12(34.3)$ & $35(100.0)$ & \multirow{4}{*}{0.39} \\
\hline & $6-10$ & $16(80.0)$ & $4(20.0)$ & $20(100.0)$ & \\
\hline & $11-15$ & $8(57.1)$ & $6(42.9)$ & $14(100.0)$ & \\
\hline & $>16$ & $6(50.0)$ & $6(50.0)$ & $12(100.0)$ & \\
\hline \multirow{3}{*}{ History of smoking } & Non smoker & $50(65.8)$ & $26(34.2)$ & $76(100.0)$ & \multirow{3}{*}{0.79} \\
\hline & Former smoker & $3(60.0)$ & $2(40.0)$ & $5(100.0)$ & \\
\hline & Present smoker & $0(0.0)$ & $0(0.0)$ & $0(0.0)$ & \\
\hline \multirow{4}{*}{$\begin{array}{l}\text { Body mass index } \\
\left(\mathrm{kg} / \mathrm{m}^{2}\right)\end{array}$} & $<18.5$ & $0(0.0)$ & $1(100.0)$ & $1(100.0)$ & \multirow{4}{*}{0.34} \\
\hline & $18.5-24.9$ & $12(57.1)$ & $9(42.9)$ & $21(100.0)$ & \\
\hline & $25-29.9$ & $22(73.3)$ & $8(26.7)$ & $30(100.0)$ & \\
\hline & $>30$ & $19(65.5)$ & $10(34.5)$ & $29(100.0)$ & \\
\hline \multirow{4}{*}{$\begin{array}{l}\text { Systolic blood pressure } \\
(\mathrm{mmHg})\end{array}$} & $<90$ & $1(100.0)$ & $0(0.0)$ & $1(100.0)$ & \multirow{4}{*}{0.15} \\
\hline & $90-120$ & $23(76.7)$ & $7(23.3)$ & $30(100.0)$ & \\
\hline & $121-139$ & $14(50.0)$ & $14(50.0)$ & $28(100.0)$ & \\
\hline & $\geq 140$ & $15(68.2)$ & $7(31.8)$ & $22(100.0)$ & \\
\hline \multirow{4}{*}{$\begin{array}{l}\text { Diastolic blood pressure } \\
(\mathrm{mmHg})\end{array}$} & $<60$ & $6(75.0)$ & $2(25.0)$ & $8(100.0)$ & \multirow{4}{*}{0.67} \\
\hline & $60-80$ & $33(62.3)$ & $20(37.7)$ & $53(100.0)$ & \\
\hline & $81-90$ & $10(76.9)$ & $3(23.1)$ & $13(100.0)$ & \\
\hline & $>90$ & $4(57.1)$ & $3(42.9)$ & $7(100.0)$ & \\
\hline
\end{tabular}

\section{Discussion}

Diabetic patients are predisposed to various complications, one of which is diabetic nephropathy $[6,7,8]$. This study was conducted to investigate the prevalence of microalbuminuria in diabetic patients presenting at the Bamenda Regional Hospital.

The overall prevalence of microalbuminuria in diabetic patients was $34.6 \%$. These results are commensurate to previous findings in most developing countries $[8,9,12]$. In one of such studies, MA was observed in $34.48 \%$ patients with type 1 and $28.33 \%$ in patients with type 2 diabetes mellitus respectively [8]. Varied results have also been reported in diabetics in sub-Saharan Africa. Choukem et al reported a prevalence of 31\% [13], Sobngwi et al. reported $53.1 \%$ [3]. Elbagir et al. reported a 22\% [14] Eghan et al. reported $43.1 \%$ [15], and Lutale et al. reported $10.1 \%$ [16]. This variation can be attributed to factors such as differences in the populations (difference in ethnicity), definitions of MA, method of urine collection/storage, methods of measurement of MA, and sample size [12,17].

While there was no significant difference in the occurrence of MA between male and female diabetic patients in the present study, majority of participants who presented with 
MA were females $(21,25.9 \%)$ while only 7 out of $22(8.65)$ of males had MA. In a similar study, Khan et al., [18] did not find any significant association between MA and gender, but reported a higher prevalence in females. Result of the present study is however contrary to some previous studies which observed higher prevalence of MA in females than in males $[15,19]$. The high prevalence in females in this study may be as a result of the high percentage of females included in the study. Interestingly, none of the diabetic patients below the age of 40 years had MA; however, there was no significant difference in the occurrence of MA with respect to age $(\mathrm{P}>0.05)$. This result is commensurate to that of Bhoomika et al. [5] and contrary to Khudhair et al. and Ghosh et al. [12, 17].

Although MA was more common $(6,50 \%)$ in individuals with a duration of diabetes of more than 16 years than those with duration less than 16 years, this difference was not statistically significant. These results are contrary to previous studies which reported significant correlation between microalbuminuria and duration of diabetes [11, 20]. While some previous studies did not find any association between duration of diabetes and MA [4, 12]. This discrepancy may be due to insufficient patient medical history. In many African countries, diabetes may go undetected for long periods probably due to less frequent hospital visits and newly diagnosed cases of diabetes may present with wellestablished complications [12]. It has been established that during the first five years of diabetes microalbuminuria is rarely found [20] and early diagnosis of MA may be beneficial in preventing the progression to $\mathrm{DN}$ and other overt complications [20]. Interesting, 12 (42.85\%) of patients with MA had a duration of diabetes of $\leq 5$ years, implying that these cases could benefit from screening programme. A majority of diabetic patients in this study were also hypertensive that is $63(77.8 \%)$ and $23(36.5 \%)$ of the hypertensive patients had microalbuminuria. No statistically significant relationship was found between hypertension and MA in this study. Agreeing with Eghan et al. [15] and contrary to reports by Khudhair [12] which showed a significant correlation. The variation may be as a result of small sample size of the present study.

There was no statistically significant relationship between microalbuminuria and BMI in the present study. But a majority of those with microalbuminuria had a BMI of $\geq 25 \mathrm{~kg} / \mathrm{m}^{2}$. Previous reports by Parchwani et al., Lutale et al. and Ghosh et al. revealed significant relations between MA and BMI [8, 16, 17] while Khudhair et al. [12] reported no significant correlation. Furthermore, no statistical correlation was recorded in the present study between microalbuminuria and systolic/diastolic blood pressure. This is contrary to the findings of Ghosh et al. [13]. Similar results have been reported by studies [16].

\section{Conclusion}

Results of this study showed a high prevalence $(34.6 \%)$ of $\mathrm{MA}$ in the diabetic population presenting at the Bamenda
Regional Hospital. Consequently, measures of glycemic control should be enhanced in this population to prevent the progression to macroalbuminuria and ESRD which present with debilitating outcome.

\section{References}

[1] World Health Organization. 1999. "Diabetes and classification of diabetes mellitus, part 1". pg 2

[2] E. Loghmani, "Diabetes mellitus: type 1 and type 2" chapter 14 in, Stang, J. Story M (eds). Guidelines for Adolescent Nutrition Services (2005) pg167 assessed on 24-11-2015 URL:http://www.epi.umn.edu/let/pubs/adol_book.shtm

[3] E. Sobngwi, J. C. Mbanya, E. N. Moukouri, K. B. Ngu "Microalbuminuria and retinopathy in a diabetic population of Cameroon” Diabetes Res. Clin. Pract, 1999, 44(3), 191-6.

[4] B. Vujičić, T. Turk, Z. Crnčević-Orlić, G. Đorđević, S. Rački, "Diabetic Nephropathy" In Tech 2012. http://dx.doi.org/10.5772/50115. Accessed on 10/12/2015.

[5] P. Bhoomika, H. A. Dadhania-Amit, A. D. Gauravi "Study of microalbuminuria in diabetes type 2 patients as a marker of morbidity". IJBAR, 2012, 3(10), 763.

[6] T. Zelmanovitz, A. F. Gerchman, P. S. Balthazar, C. S. Fúlvio, J. Thomazelli, D. Matos, L. H. Canani "Diabetic nephropathy. Diabetology \& Metabolic Syndrome" 2009, 1(10). $\mathrm{http}: / / \mathrm{www} . d m s j o u r n a l . c o m /$ content/1/1/10 accessed on $10 / 12 / 2015$

[7] W. Sheldon, P. A. Tobe, D. McFarlane, M. Naimark "Microalbuminuria in diabetes mellitus". CMAJ, 2002, 167 (5), 499-502.

[8] D. Parchwani, S. P. Singh "Microalbuminuria in diabetes patients: Prevalence and putative risk factors" Nat. J. Com. Med, 2011, 2(1), 126.

[9] A. Abdalkader, H. Faris Al Lami "Prevalence and determinants of microalbuminurea among type 2 diabetes mellitus patients, Baghdad, Iraq". Saudi J. Kidney Dis. Transpl, 2013, 27, 348-55.

[10] M. M. Lloyd, J. Kuyl, H. Van Jaarsveld "Evaluation of point of care test for detecting microalbuminuria in diabetic patients". S. Afr. Fam. Pract, 2011, 53(3), 281-286

[11] J. J. Noubiap, J. Naidoo, A. P. Kengne "Diabetic nephropathy in Africa: A systematic review", World J. Diabetes, 2015, 10, 6(5). 759-773.

[12] S. A. Khudhair "Prevalence of Microalbuminuria In Diabetic Patients In Al-Najaf City. Kufa” Med. Journal, 2009, 12(1), 462.

[13] S. P. Choukem, E. Sobngwi, P. Boudou, L. S. Fetita, R. Porcher, I. Fidaa, B. Blondeau, P. Vexiau, F. Mauvais-Jarvis, F. Calvo J. F. "Gautier " $\beta$ - and $\alpha$-Cell Dysfunctions in Africans with Ketosis-Prone Atypical Diabetes During Near-Normoglycemic Remission” Diabetes Care, 2013, 36 (1), 118-123.

[14] M. N. Elbagir, M. A. Eltom, H. Rosling, C. Berne "Glycaemic Control of Insulin-Dependent Diabetes Mellitus in Sudan: Influence of Insulin Shortage" Diabetes Res. Clin. Pract, 1995, 30 (1), 43-52. 
[15] B. A. Eghan Jr, M. T. Frempong and M. Adjei-Poku "Prevalence and predictors of microalbuminuria in patients with diabetes mellitus: a cross-sectional observational study in Kumasi, Ghana” Ethnicity \& Disease, 2007, 7(1), 726-730.

[16] K. Luthale, J. Joy, T. Hrafenkell, A. G. Zulfiqarali, V. Kåre "Microalbuminuria among Type 1 and Type 2 diabetic patients of African origin in Dar Es Salaam, Tanzania", BMC Nephrol, 2007, 8(2). http://www.biomedcentral.com/1471-2369/8/2.

[17] S. Ghosh, I. Lyaruu, K. Yeates "Prevalence and factors associated with microalbuminuria in type 2 diabetic patients at a diabetes clinic in northern Tanzania". Afr. J. Diabetes Med, 2012, 20(2), 43.
[18] P. Khan, M. Khan, W. Khan, A. Aziz and A. Ahad "Relationship of Glycemic Control with Prevalence of Microalbuminuria in Diabetic Patients" Gomal J. Med. Sci, 2012, 10(2), 201-204.

[19] M. Sigdel, N. Rajbhandari, S. Basnet, A. Nagila, P. Basnet, B. K. Tamrakar "Microalbuminuria amongtype 2 diabetes mellitus patients in Pokhara, Nepal" Nepal Med. Coll. J, 2008, $10,242-5$.

[20] M. T. Pruijm, G. Madeline, W. F. Rieson, M. Burnier, P. Bovet "Prevalence of microalbuminuria in the general population of Seychelles and strong association with diabetes and hypertension independent of renal markers" J. Hypertens, 2008, 26, 871-7. 